auf $C_{s}$ erniedrigen. Im IR-Spektrum der festen Säure wurden die zweifach-entarteten Schwingungen $\nu_{4}, \nu_{5}$ und $\nu_{6}$ jeweils aufgespalten (1150 und 1200, 4,20 und 540,1000 und $1090 \mathrm{~cm}^{-1}$ ), was für eine Erniedrigung der Symmetrie von $C_{3 v}$ auf $C_{s}$ bzw. $C_{1}$ hindeutete. Auf Grund des IR-Spektrums läßt sich jedoch zwischen den zwei letzten Gruppen nicht entscheiden und für eine Lage-Symmetrie kommt die sonst mögliche Gruppe $C_{3}$ nicht in. Betracht, sondern nur entweder $C_{s}$ oder $C_{1}$.
Die Valenzschwingung $\nu \mathrm{PH}$ absorbiert bei $2440 \mathrm{~cm}^{-1}$, also bei fast derselben Wellenzahl wie das hydratisierte Yttriumphosphit. Diese Absorption ist ein weiterer Beweis für das Vorhandensein eines Anions mit den Liganden $\mathrm{HPO}_{3}{ }^{2}{ }^{\ominus}$, da im Falle des Anions $\mathrm{H}_{2} \mathrm{PO}_{3}{ }^{\ominus}$ die $\nu \mathrm{PH}$ höher liegen müßte.

Ein weiteres Studium dieser Verbindungen (auch von Sc und $\mathrm{La}$ ) ist im Hinblick auf die Möglichkeit der optischen Isomerie vorgesehen.

\section{N-Halogen-imidoschwefeloxidifluoride, $\mathrm{X}-\mathrm{NSOF}_{2}$}

$\mathrm{N}$-Halogen-imidosulphuroxidifluorides, $\mathrm{X}-\mathrm{NSOF}_{2}$

\section{KonRad SEPpelt und Wolfgang Sundermeyer}

Anorganisch-Chemisches Institut der Universität Heidelberg (Z. Naturforsch. 26 b, 65--66 [1971] ; eingegangen am 11. November 1970)

Mit den kürzlich von uns dargestellten Verbindungen Trimethylsilyl-imidoschwefeloxidifluorid, $\left(\mathrm{CH}_{3}\right)_{3} \mathrm{Si}$ $\mathrm{NSOF}_{2}$ (1) und Quecksilber-bis(imidoschwefeloxidifluorid), $\mathrm{Hg}\left(\mathrm{NSOF}_{2}\right)_{2}(2)^{1}$ erhielten wir Schlüsselsubstanzen, um präparativ günstig unter anderem zu den $\mathrm{N}$-Halogenimidoschwefeloxidifluoriden, X-NSOF, zu gelangen, von welcher Reihe die $\mathrm{N}-\mathrm{Br}$ - und $\mathrm{N}-\mathrm{J}$-Verbindung mit den bisher bekannten Methoden ${ }^{2,3}$ vergeblich darzustellen versucht worden waren.

Läßt man auf eine Lösung von 2 in Methylenchlorid Jodchlorid im Molverhältnis $1: 2$ bei Zimmertemperatur einwirken, filtriert die Lösung und destilliert das Lösungsmittel ab, so erhält man mit fast quantitativer Ausbeute N-Jod-imidoschwefeloxidifluorid (3).

$$
\underset{2}{\mathrm{Hg}}\left(\mathrm{NSOF}_{2}\right)_{2}+2 \mathrm{JCl} \rightarrow \underset{3}{\mathrm{~J}}-\underset{\mathrm{F}}{\mathrm{N}}=\underset{\mathrm{F}}{\mathrm{S}}=\mathrm{O}+\mathrm{HgCl}_{2} .
$$

3 bildet tiefgelbe Kristalle, die nach eine: Sublimation bei $25^{\circ} \mathrm{C}$ einen Schmelzpunkt von $55^{\circ} \mathrm{C}$ haben (Dampfdruck 5 Torr $/ 25^{\circ} \mathrm{C}$ ). Neben der Elementaranalyse wurde das Massenspektrum zur Identifizierung herangezogen $\left[\mathrm{m} / e=227\left(\mathrm{JNSOF}_{2} 20 \%\right), 189\right.$ (JNSO $4 \%), 141$ (JN 16\%), 127 (J 100\%), $100\left(\mathrm{NSOF}_{2} 2 \%\right)$, 81 (NSOF 4\%), 67 (SOF 70\%) und 62 (NSO 10\%); $70 \mathrm{eV}]{ }^{4}$. Sowohl das IR-Spektrum [Schmelze, 1370 (sst, $\nu \mathrm{SO}), 1180$ (sst, $\nu \mathrm{SN}$ ) und $790 \mathrm{~cm}^{-1}$ (sst, $\left.\left.\nu \mathrm{SF}\right)\right]$, als auch das ${ }^{19} \mathrm{~F}$-NMR-Spektrum $(\delta=-50,0 \mathrm{ppm}$ in $\mathrm{CH}_{2} \mathrm{Cl}_{2}$ mit $\mathrm{CFCl}_{3}$ als internem Standard bei $\left.20^{\circ} \mathrm{C}\right)^{4}$ deuten auf die angenommene Struktur hin. Die UV-Absorption liegt bei $3150 \AA$ in $\mathrm{CH}_{2} \mathrm{Cl}_{2}$.

N-Brom-imidoschwefeloxidifluorid, $\mathrm{Br}^{-\mathrm{NSOF}_{2}}$ (4), wird durch Reaktion von 2 mit Brom bei Raumtemperatur und unter Rühren im Autoklaven (ca. 12 Stdn.)

Sonderdruckanforderungen an Prof. Dr. W. Sundermeyer, Anorganisch-Chemisches Institut der Universität, D-6900 Heidelberg 1, Im Neuenheimer Feld 7.

1 K. Seppelt u. W. Sundermeyer, Angew. Chem. 82, 931 [1970] ; Angew. Chem. Int. Ed. 9, 905 [1970].

2 J. K. Ruff, Inorg. Chem. 5, 1787 [1966].

3 K. Seppelt, Dissertation, Universität Heidelberg 1970. mit quantitativer Ausbeute erhalten. Durch einen geringen Überschuß an 2 und das Weglassen eines Lösungsmittels kann 4 sofort rein und bromfrei erhalten werden. Es ist eine rote Flüssigkeit: Schmp. $-43^{\circ} \mathrm{C}$, Sdp. $50^{\circ} \mathrm{C}$ (extrapoliert aus der Dampfdruckkurve). Das Massenspektrum zeigt Peaks bei $m / e=179,181$ $\left(\mathrm{BrNSOF}_{2} \quad 69 \%\right), \quad 141,143$ (BrNSO $\left.19 \%\right), 100$ $\left(\mathrm{NSOF}_{2} 32 \%\right)$, 93, 95 (BrN 100\%), 79, 81 (Br 35\%) und 67 (SOF 63\%); $70 \mathrm{eV}$. Während die UV-Absorption bei $2750 \AA$ liegt, findet man im IR-Spektrum (gasförmig) Banden bei 1404 (sst, $v$ SO), 1163 (sst, $\nu \mathrm{SN})$ und $817 \mathrm{~cm}^{-1}$ (sst, $\left.\nu \mathrm{SF}\right) .{ }^{19} \mathrm{~F}-\mathrm{N} M R$-Spektrum: $\delta=-44,3 \mathrm{ppm}$ gegen $\mathrm{CFCl}_{3}$, intern bei $20^{\circ} \mathrm{C}$.

Völlig analog zur Darstellung von 4 erhält man das N-Chlorimidoschwefeloxidifluorid, Cl-NSOF ${ }_{2}(5)$, aus 2 und Chlor in präparativ günstigerer Weise als bisher beschrieben ${ }^{2}$. Die Substanz wurde an Hand der bekannten Massen-, IR- und UV-Spektren identifiziert. Der Schmelzpunkt wurde bei $-89^{\circ} \mathrm{C}$ gefunden; Sdp. $19,8{ }^{\circ} \mathrm{C}$. Abweichend von l.c. ${ }^{2}$ zeigt das ${ }^{19} \mathrm{~F}$-NMRSpektrum einen $\delta$-Wert von $-40,4 \mathrm{ppm}$ gegen $\mathrm{CFCl}_{3}$, intern bei $20^{\circ} \mathrm{C}$. Die Absorption im UV liegt bei $2230 \AA \AA$ in Cyclohexan.

Mit Fluor reagiert 2 im Autoklaven bei $-193{ }^{\circ} \mathrm{C}$ unter Bildung geringster Mengen von N-Fluor-imidoschwefeloxidifluorid, F-NSOF ${ }_{2}$, einer hochexplosiven Substanz (vgl. l. c. ${ }^{2}$, deren Siedepunkt bei ca. $-10{ }^{\circ} \mathrm{C}$ (extrapoliert aus mehreren Dampfdrucken) und deren IR-Absorptionen (gasförmig) bei 1409 (sst, $\nu \mathrm{SO}), 1122$ (sst, $\nu \mathrm{SN})$ und $848 \mathrm{~cm}^{-1}$ (sst, $\nu \mathrm{SF}$ ) liegen. Bei Darstellungsversuchen bei Raumtemperatur erhält man nur $\mathrm{HgF}_{2}, \mathrm{~N}_{2}, \mathrm{SOF}_{2}$ und wenig $\mathrm{SO}_{2} \mathrm{~F}_{2}$; bei $-78{ }^{\circ} \mathrm{C}$ zusätzlich viel $\mathrm{SOF}_{4}$.

Präparativen Vorteil bietet der hier beschriebene Weg auch zur Darstellung des Imidoschwefesoxidifluorids, H-NSOF ${ }_{2}(6)$ (vgl. l. c. ${ }^{5}$ ). 2 reagiert mit Chlorwasserstoff ohne Lösungsmittel im Autoklaven bei Raumtemperatur gemäß

$$
\underset{2}{\mathrm{Hg}} \underset{2}{\left(\mathrm{NSOF}_{2}\right)_{2}}+2 \mathrm{HGl} \rightarrow \underset{6}{2} \underset{\mathrm{HSOF}}{\mathrm{H}}+\mathrm{HgCl}_{2} .
$$

4 Für die Aufnahme der Massenspektren danken wir Herrn Dr. H. SEIDL, Badische Anilin- und Sodafabrik, für die Aufnahme der ${ }^{19} \mathrm{~F}$-NMR-Spektren den Herren Prof. Dr. E. Fluck, Stuttgart, und Doz. Dr. H. P. Latscha, Heidelberg, sehr herzlich.

5 G. W. Parshall, R. Cramer u. R. E. Foster, Inorg. Chem. 1, 677 [1962]. 
6 ist eine wasserklare Flüssigkeit: Schmp. $-33{ }^{\circ} \mathrm{C}$, Sdp. $43{ }^{\circ} \mathrm{C}^{5}$. Die Identifizierung erfolgte durch das Massenspektrum $\left[\mathrm{m} / \mathrm{e}=101\left(\mathrm{HNSOF}_{2} 58 \%\right), 86\right.$ $\left(\mathrm{SOF}_{2} 22 \%\right), 85\left(\mathrm{HNSF}_{2} 36 \%\right), 82$ (HNSOF 32\%), 81 (NSOF 34\%), 67 (SOF 99\%), 63 (HNSO 100\%), 48 (SO 90\%) und 46 (SN 84\%); $70 \mathrm{eV}$, durch das IRSpektrum [gasförmig, 3418 (st, $v \mathrm{NH}), 1448$ (sst, $\nu \mathrm{SO}$ ), 1408 (sst, $\delta \mathrm{NH}), 1210$ (sst, $\nu \mathrm{SN}), 931(\mathrm{~m}, \gamma \mathrm{NH})$, 848 (sst, $\nu_{\mathrm{as}} \mathrm{SF}$ ) und $818 \mathrm{~cm}^{-1}$ (sst, $\nu_{\mathrm{s}} \mathrm{SF}$ )] und durch das ${ }^{19} \mathrm{~F}$-NMR-Spektrum: $\delta=-55,5 \mathrm{ppm}$ gegen $\mathrm{CFCl}_{3}$, intern bei $20^{\circ} \mathrm{C}$. - Analog wurde auch aus 2 und Deuteriumchlorid das N-Deuterium-imidoschwefeloxidifluorid, D-NSOF 2 , dargestellt und zum Vergleich mit
6 das Massenspektrum und das IR-Spektrum aufgenommen [gasförmig, 2544 (st, $\nu \mathrm{ND}), 1433$ (sst, $\nu \mathrm{SO}$ ), 1182 (st, $\nu \mathrm{SN}$ ), 1173 (st, $\delta \mathrm{ND}$ ), 848 (st, $\nu_{\mathrm{as}} \mathrm{SF}$ ), 818 (st, $\left.v_{\mathrm{s}} \mathrm{SF}\right)$ und $\left.718 \mathrm{~cm}^{-1}(\mathrm{~m}, \gamma \mathrm{ND})\right]$.

Während polare Verbindungen wie Jodchlorid und Chlorwasserstoff auch die $\mathrm{Si}-\mathrm{N}$-Bindung in $\mathbf{l}$ zu spalten vermögen, reagieren Chlor und Brom nur unter Halogenierung der Methylgruppen. Der entstehende Halogenwasserstoff zersetzt 1 zu 6.

Der Deutschen Forschungsgemeinschaft, dem Fonds der Chemischen Industrie, den Farbenfabriken Bayer AG. und der der Badischen Anilin- und Sodafabrik danken wir für Sachmittel bzw. Chemikalien.

\section{Photochemische Darstellung von Butadien-eisen- tricarbonyl und dessen Überführung in Bis-buta- dien-eisenmonocarbonyl}

Photochemical Preparation of Butadiene Iron Tricarbonyl and its Transformation to bis-Butadiene Iron Monocarbonyl

\section{E. Koerner von Gustorf, Z. Pfajfer und F.-W. Grevels}

Max-Planck-Institut für Kohlenforschung, Abteilung Strahlenchemie, Mülheim/Ruhr

(Z. Naturforsch. 26 b, 66-67 [1971] ; eingegangen am 5. November 1970)

Kaum ein Olefin-Metallkomplex ist so eingehend untersucht worden wie das Butadien-eisentricarbonyl (1). Für seine Darstellung wird jedoch bislang nur ein Verfahren empfohlen ${ }^{1}$, das in der Umsetzung von Butadien mit $\mathrm{Fe}(\mathrm{CO})_{5}$ bei $140^{\circ} \mathrm{C}$ im Autoklaven besteht und auf die ursprüngliche Verfahrensweise der Entdecker von 1 zurückgeht ${ }^{2}$. Die Ausbeute dieses Verfahrens liegt bei $42 \%$ [bezogen auf umgesetztes $\mathrm{Fe}(\mathrm{CO})_{5}$ ] bzw. $17 \%$ [bezogen auf eingesetztes $\mathrm{Fe}(\mathrm{CO})_{5}$ ].

Im Zusammenhang mit einer systematischen Untersuchung der Photochemie von Olefin-Metallcarbonylen ${ }^{3}$ haben wir die photochemische Darstellung von 1 ausgearbeitet. Da dieses Verfahren recht einfach und ergiebiger [65\% bezogen auf eingesetztes $\mathrm{Fe}(\mathrm{CO})_{5}$ ] als das thermische ${ }^{1}$ ist, teilen wir die experimentellen Einzelheiten nachstehend mit.

Als Nebenprodukt bei dieser Reaktion haben wir das Bis-butadien-eisenmonocarbonyl (2) entdeckt ${ }^{4}$, das im Verlauf einer Photoreaktion von 1 mit Butadien entsteht. 2 läßt sich unter geeigneten Bedingungen auch direkt aus $\mathrm{Fe}(\mathrm{CO})_{5}$ ohne Isolierung von 1 gewinnen ${ }^{4}$.

Sonderdruckanforderungen an Dr. E. A. KoERNER voN Gustorf, MPI f. Kohlenforschung, Abteilung Strahlenchemie, D-4330 Mülheim/Ruhr, Stiftstr. 34-36.

1 R. B. KInG, Organometallic Syntheses 1, 128 [1965], Academit Press, New York.

2 H. Reihlen, A. Gruhl, G. von Hessling u. O. Pfrengle, Liebigs Ann. Chem. 482, 161 [1930].

3 E. Koerner von Gustorf u. F.-W. Grevels, Fortschr. chem. Forsch. 13, 366 [1969].

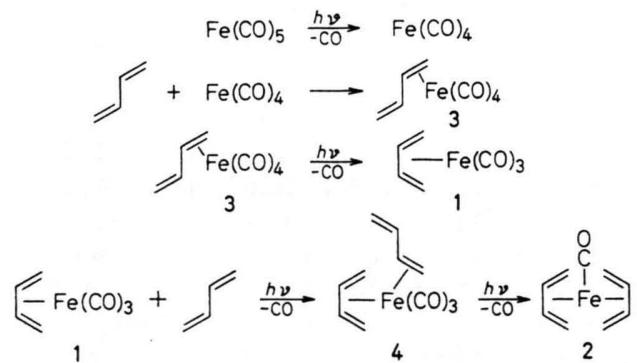

2 ist der erste Vertreter der Olefin-Eisenmonocarbonyle. Die einzelnen Schritte der Bildung von 1 und 2 sind in $\mathrm{Gl}$. (1) - (4) wiedergegeben.

Reaktion (1) haben wir blitzspektroskopisch nachgewiesen ${ }^{3,5}$, (2) und (3) sind durch eine Vielzahl von Beispielen belegt ${ }^{3,6}$. Butadien-eisentetracarbonyl (3) wandelt sich thermisch unter unseren Reaktionsbedingungen nur langsam in 1 um $^{7}$, (3) ist demnach eine photochemische Reaktion. 4 haben wir noch nicht isoliert.

\section{Arbeitsvorschriften}

Butadien-eisentricarbonyl (1): $49 \mathrm{~g} \quad(0,25 \mathrm{Mol})$ $\mathrm{Fe}(\mathrm{CO})_{5}$ und $54 \mathrm{~g}$ (1 Mol) flüssiges Butadien in $150 \mathrm{ml}$ Benzol werden unter Argon in einer Tauchlampenapparatur aus Solidex-Glas bei $20{ }^{\circ} \mathrm{C}$ bis zur Entwicklung von $0,5 \mathrm{Mol} \mathrm{CO}$ mit einem Quecksilberhochdruckbrenner Philips HPK $125 \mathrm{~W}$ bestrahlt. Durchschnittlich braucht man dazu zwei Tage. Aus der Lösung austretendes Butadien wird an einem mit festem $\mathrm{CO}_{2} /$ Aceton beschickten Kühlfinger kondensiert und fließt zurück. Die belichtete Lösung wird schnell durch ein Faltenfilter filtriert, Benzol und Reste von $\mathrm{Fe}(\mathrm{CO})_{5}$ werden im Wasserstrahlvakuum abgezogen und der Rückstand

${ }^{4}$ E. Koerner von Gustorf, J. Buchkremer, Z. Pfajfer u. F.-W. Grevels, Angew. Chem., im Druck.

5 E. Koerner von Gustorf, N. Harrit u. H. Hermann, noch unveröffentlicht.

6 E. Koerner von Gustorf u. J. Hogan, Tetrahedron Letters [London] 1968, 3191.

7 H. D. Murdoch u. E. Weiss, Helv. chim. Acta 45, 1156 [1962]. 